\title{
Graphene: Fundamentals and functionalities
}

\author{
Weijie Lu, Patrick Soukiassian, and John Boeckl, Guest Editors
}

\begin{abstract}
Graphene came to the forefront in the nanosciences in the early 2000s, in particular, when high-quality graphene with atomic thickness and two-dimensional extension in the micrometer range was isolated and the resulting novel electronic properties were demonstrated. Graphene has two unique features: lateral size up to tens of micrometers or larger and quantum confinement in an atomically thin sheet. It provides an excellent platform for exploring novel material properties, designing new materials, and enhancing material performance. Now, after extensive research for nearly a decade, graphene research has moved well beyond electronic applications and has begun to extend into a wide variety of disciplines. This expanded issue of MRS Bulletin is focused on graphene and consists of 20 articles and three commentaries that collectively address the major impact of graphene on materials science, highlight the newest advances, discuss challenging issues, explore applications, and reveal future directions.
\end{abstract}

\section{Introduction}

Nanomaterials have become an important part of materials research over the past 30 years. Carbon nanomaterials, in particular, have been at the forefront of nanomaterials developments in recent decades. Fullerenes $\left(\mathrm{C}_{60}\right)$ - discovered by Smalley et al. ${ }^{1}$ in 1985 - became one of the first recognized nanomaterials and played an important role in the early development of nanoscience and nanoengineering. In the 1990s, carbon nanotubes (CNTs), discovered by S. Iijima and colleagues, became one of the most intensively investigated nanomaterials. ${ }^{2}$ More recently, graphene has gained significant attention and has become one of the most widely investigated materials. ${ }^{3,4}$ Indeed, today, graphene science and technology is a broad research field.

Graphene is a single-atomic-layer honeycomb lattice of carbon atoms in an $s p^{2}$ hexagonal bonding configuration (Figure 1). It is a two-dimensional allotrope of $s p^{2}$ carbon in the form of a planar monolayer. Graphene can be considered to be the basic atomic structural unit for a wide range of carbon nanomaterials. Zero-dimensional $\mathrm{C}_{60}$ can be considered to be graphene with the introduction of a pentagonal arrangement of carbon atoms - and therefore curvature - to form a ball structure. One-dimensional carbon nanotubes (CNTs) are essentially graphene sheets rolled into cylinders with a variety of chiralities depending on the direction of rolling with respect to the hexagonal lattice. The physical properties of carbon vary widely with the allotropic form. Low-dimensional carbon nanomaterials, including graphene, $\mathrm{CNTs}$, and $\mathrm{C}_{60}$, have had a significant impact on the exploration of novel material properties, the design of new functional materials, and the enhancement of material performance.

In 2004, Novoselov et al. ${ }^{3}$ discovered that a high-quality isolated graphene sheet exhibits a strong ambipolar electric field effect with a carrier concentration of $10^{13} \mathrm{~cm}^{-2}$ and a roomtemperature mobility of $10,000 \mathrm{~cm}^{2} \mathrm{~V}^{-1} \mathrm{~s}^{-1}$. The direct observation of isolated graphene monolayers that year sparked exponentially growing interest in this material and motivated research into the properties of this two-dimensional nanomaterial. The unique electron-transport properties of two-dimensional graphene from the atomic scale to the macroscale first attracted the attention of solid-state physicists and then of materials scientists, chemists, biologist, and engineers from various disciplines. These disparate research communities began exploring ways to incorporate graphene into devices for applications to exploit the novel properties of this unique nanomaterial, as highlighted in recent review articles..$^{5-11}$

Although nanomaterials have had a significant recent impact on materials science and engineering, more conventional nanomaterials - such as quantum dots, carbon nanotubes, and oxide nanowires-lack structural integration in the scale 


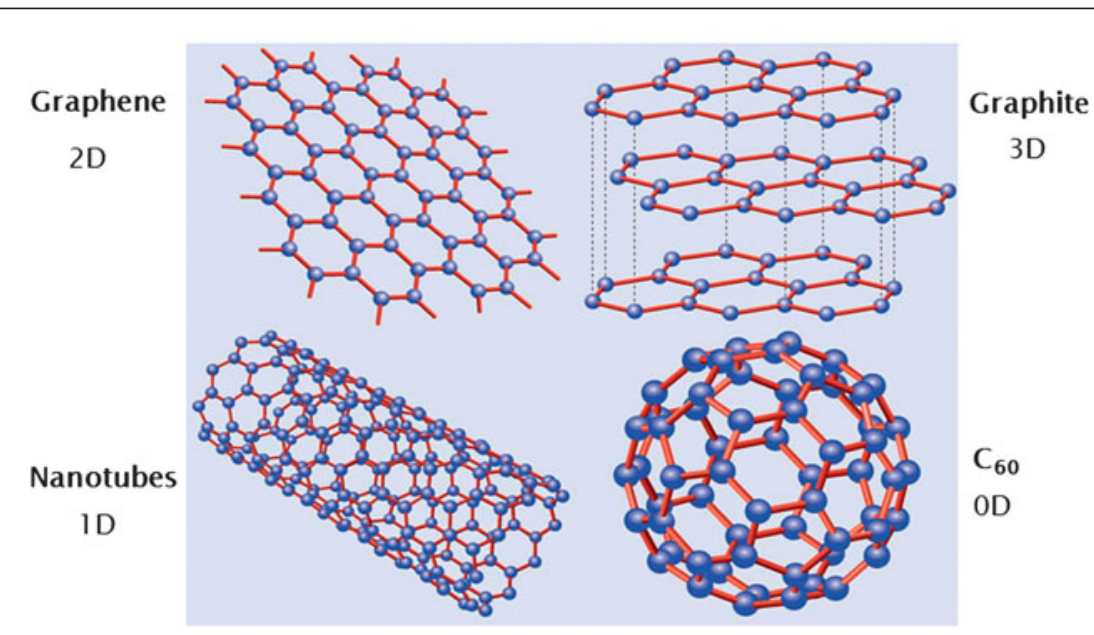

Figure 1. Allotropes of $s p^{2}$ carbon materials. ${ }^{11}$ for other nanomaterials. The large number of reports on novel properties and functionalities of graphene in recent years has demonstrated the impact of the large lateral dimensions of graphene. Further, graphene excels as both a building block and a platform for nanostructures at the micro- to macroscales. Thus, although graphene does not represent a direct answer to the challenges illustrated in Figure 2, it allows for novel approaches to avoid and overcome these issues in the multiscale integration of nanomaterials.

Graphene has clearly had a major impact on fundamental research in nanomaterials. Graphene-based two-dimensional science and technology has become a new interdisciplinary area and has impacted the landscape of materials research. After nearly a decade of intensive research, it is timely to review this rapidly

range from nanometers to micrometers. Quantum dots of controllable shape and size at the nanometer scale have been demonstrated; however, it is difficult to assemble structurally ordered quantum dots in composites at the scale of micrometers, for example. Similar challenges hold for one-dimensional CNTs and nanowires. One of the main issues is that it is difficult to measure the properties of an individual quantum dot, CNT, or other similar nanostructure. Currently, there is little understanding of the fundamentals of materials growth at multiple hierarchical scales. The challenges of "bottom-up" fabrication in nanoscience and nanotechnology have remained for decades. The consequence is that only limited success has been achieved for the more conventional nanomaterials in fabricating multiscale hierarchical nanostructures able to yield large material volumes to complement manufacturing technologies. Figure 2 illustrates two fundamental scale-integration challenges in traditional nanostructured materials: (1) understanding the growth process from initial chemical reactions at the atomic and molecular scales toward building nanoscale materials, including shape, size, and uniformity of nanomaterials and assembling structures, and (2) developing materials processing from the nanoscale to the micrometer scale and beyond toward high-volume production for specific applications.

Compared to other nanomaterials, graphene is structurally unique in that, whereas the lateral dimensions of graphene are up to tens of micrometers or larger, the thickness is at the atomic scale. Although the growth mechanism of graphene from the atomic scale to the macroscale is still the subject of investigation, the large lateral dimensions possible with graphene have allowed for the direct measurements of physical properties on a single nanostructure. Therefore, graphene has provided a practical and easy approach to investigate intrinsic nanoscale properties, not limited to overall average properties as developing field, summarize the state of the art, and explore future research directions. This expanded issue of MRS Bulletin includes 20 articles and three commentaries by noted researchers in this field and provides broad coverage of the fundamentals and functionalities of graphene.

\section{Fundamentals \\ Materials developments}

The Georgia Institute of Technology group led by Walter de Heer has been a leader in developing epitaxial graphene on $\mathrm{SiC}$ structures for electronics applications. ${ }^{4} \mathrm{SiC}$ is annealed in a vacuum or in an argon atmosphere at high temperature. Silicon near the surface of the $\mathrm{SiC}$ lattice desorbs, and free carbon on the surface transforms into graphene with a hexagonal lattice structure. Whereas mechanical exfoliation of graphene (initially production of high-quality graphene for exploring and discovering novel properties and phenomena, growth of epitaxial graphene on $\mathrm{SiC}$ represents a reliable fabrication technique for electronic devices on an industrial scale ${ }^{4}$ (also see the articles in this issue by Ruan et al. and Nyakiti et al.). Another promising growth technique for graphene is surface graphitization on metals by catalysis using chemical vapor deposition (CVD) (see the article in this issue by Bartelt and McCarty). Once grown, demonstrated by Novoselov et al. ${ }^{3}$ ) allows for laboratory-scale 
the graphene can be transferred onto an insulating substrate for device applications. Although synthesis of device-grade graphene by CVD on an industrial scale is already under development, understanding of the growth mechanisms of metal catalytic surface graphitization will enable progress toward this end.

Chemical exfoliation from graphite powders through the formation of graphite oxides by oxidation of graphite in solution is another well-studied fabrication technique. This solutionbased process holds particular promise because of its low cost, high throughput, chemical versatility, and scalability to large quantities. Furthermore, solution-processed graphene can be seamlessly integrated with other nanomaterials or polymers to yield composites for a wide array of applications such as energy conversion and storage, catalysis, electronics, and highstrength materials (see the article in this issue by Jaber-Ansari and Hersam).

Graphene produced by these available techniques is far from perfect. Several of the articles in this issue discuss defects, modifications, and characterization of graphene. Huang et al. review transmission electron microscopy (TEM) of graphene, which can reveal many structural details, such as layer thickness and stacking, grain sizes and orientations, static deformations, topological defects, vacancy atomic configurations, grain boundary structures, and dislocations, and provide microscopic access to electronic configurations. Pantelides et al. discuss intrinsic defects, such as vacancies, interstitials, and line defects, and their potential role in transport and other properties. In addition, they describe impurities and adsorbates that can act as dopants to enhance carrier densities, controlling $n$ - and $p$-type conduction, and can serve as reactive sites for catalytic and sensor applications. Wong et al. explain the effectiveness of scanning tunneling microscopy (STM) and scanning tunneling spectroscopy (STS) measurements of epitaxial graphene on $\mathrm{SiC}$ for the characterization of topological and electronic properties of graphene on the local scale. STM and STS can be used to determine the number of graphene layers, defects, rotational disorder between graphene layers, and mismatch between graphene and its substrate, as well as to provide the local electronic characterization of graphene. Synchrotron radiation is another effective tool for characterizing a wide variety of properties, including transport for graphene-SiC interface doping, bandgap formation, and ohmic contact formation (see the article in this issue by Hicks and Conrad).

Further, Starke et al. demonstrate the manipulation of the electronic structure of epitaxial graphene by transfer doping and atomic intercalation. Hydrogen atoms migrate under graphene on $\mathrm{SiC}$, passivate the underlying $\mathrm{SiC}$ layer, and decouple the graphene from the substrate. In this way, large-scale, homogeneous, quasi-free-standing graphene layers can be obtained. By controlling the amount of intercalated germanium, two symmetrically doped — $n$ - and $p$-type — phases can be stabilized.

\section{Fundamental understanding of graphenization}

Graphite is a well-studied material that is widely applied in industry. The growth techniques for producing high-quality graphite are mature, and the fundamental issues of graphite are well understood. Graphene could be viewed as a material formed from graphite by dimensional reduction. As the quality of graphene is one of main concerns for applications, comparison with fundamental graphite issues should be helpful in the development of graphene materials.

Graphitization is a disorder-order transformation in hexagonal carbon networks that is a thermally activated kinetic process with a well-defined and physically significant activation energy. ${ }^{12}$ As the structures of the hexagonal networks become ordered and defects are reduced, the interlayer distance in graphite approaches $3.35 \AA$. Graphitization is thus a threedimensional structural evolution process, with the degree of graphitization characterized by the interlayer distance.

In contrast to graphitization, where the fundamental issues are well understood, graphenization is not a well-defined term. ${ }^{14}$ Currently, graphene is obtained "as-grown" by a "trial-anderror" approach, and structural characterizations are usually applied to confirm the presence of graphene. Graphenization can be defined as a process to form two-dimensional hexagonal networks consisting of $s p^{2}$ carbon atoms, transform them from disordered to ordered, reduce structural defects, and expand the lateral dimensions. The quantitative description for each step needs to be established, and many issues related to graphenization are under investigation and have not yet been addressed. Table I summarizes our current understanding of the fundamental issues for graphene by comparison with those for graphite.

The quality of graphene is not always evaluated and is rarely mentioned in the majority of published studies. Thus, a standard for the quality of graphene needs to be defined, and an accepted evaluation method for graphene needs to be determined. The carrier mobility in epitaxial graphene synthesized on $\mathrm{SiC}$ by surface decomposition and metal catalytic CVD is much lower than that measured in graphene that is mechanically exfoliated from natural graphite (see the article in this issue by Avouris and $\mathrm{Xia}$ ). Higher-quality graphene is obtained as the interface states and defects are reduced. It is extremely difficult to obtain graphene with high structural purity by chemical exfoliation. Many material quality issues related to graphene produced by chemical exfoliation need to be resolved, including control of the size, number of layers, uniformity, purity, and type and density of defects. In addition, scientific understanding is needed for various factors, including graphene chemical exfoliation efficiency, graphene oxide reduction mechanism, active sites and density for functionalization, surface energetic distribution, activation energy and distribution, functional-group effects, surface chemistry, matrix effects, and interface and interphase interactions.

All of these structural parameters are associated with the quality of graphene produced. Another aspect pertains to graphene functionalities, such as carrier mobility, surface area, optical response, and thermal conductivity, which could be applied for the evaluation of graphene quality. These aspects need to be addressed for the successful development of graphenebased fabrication techniques. Material quality is essential for successful applications and commercialization, and a generally 
Table I. Fundamental issues of graphene in comparison with those of graphite.

\begin{tabular}{|c|c|c|}
\hline & Graphitization & Graphenization \\
\hline Definition & Clearly defined & $\begin{array}{l}\text { Not well-defined; low-dimensional } \\
\text { structures formed }\end{array}$ \\
\hline Activity energy & Known & Ongoing research \\
\hline Pressure effect & Yes, but not fully understood & Yes, but the mechanism is unknown \\
\hline $\begin{array}{l}\text { Temperature effect (non-metal } \\
\text { catalytic process) }\end{array}$ & $>2000^{\circ} \mathrm{C}$ & $1300-1700^{\circ} \mathrm{C}$ \\
\hline $\begin{array}{l}\text { Temperature effect (metal catalytic } \\
\text { process) }\end{array}$ & $<1000^{\circ} \mathrm{C}$ & $<1000^{\circ} \mathrm{C}$ \\
\hline Kinetics & Known & Unknown \\
\hline $\begin{array}{l}\text { Main structural characterization } \\
\text { techniques }\end{array}$ & X-ray diffraction & Microscopy and Raman spectroscopy \\
\hline Starting materials & Solids & Usually gases or SiC \\
\hline Mechanism at the atomic scale & Diffusion & Diffusion likely, not clear \\
\hline Evaluation & Degree of graphitization & Degree of graphenization \\
\hline Structure-property relationship & Known & Ongoing research \\
\hline
\end{tabular}

The impact of graphene has moved well beyond fieldeffect transistors and expanded rapidly into many other applications in electronics. For example, with intraband and free-carrier transitions in the microwave and terahertz regimes, it is expected that graphene will improve the performance of conventional electronic and photonic devices operating in the terahertz frequency range (see the article in this issue by Otsuji et al.).

Graphene exhibits very long spin diffusion lengths in the 100- $\mu \mathrm{m}$ range, much longer than those in conventional metals and semiconductors. accepted method for evaluating the quality of graphene needs to be established by identifying key structural characterization techniques and functionality parameters.

Many fundamental issues remain to be addressed for graphene. The growth mechanisms of two-dimensional structures at the atomic scale are a new challenge in materials research. Further, chemical kinetics is often the key for reducing structural defects in a growth process. Various kinetics issues in graphene growth remain to be addressed, such as activation energy, reaction order, diffusion mechanisms, and barriers. A detailed understanding of these fundamental issues is critical for the improvement of graphene quality and the development of new growth techniques.

\section{Multiple functionalities of graphene}

The atomic thickness of graphene yields novel material properties, and the large lateral dimensions that can be achieved with graphene make it easy to process and handle. This advantage of scale has enabled many unique properties of graphene to be discovered. For example, exfoliated graphene has shown ultrahigh intrinsic carrier mobility, up to $200,000 \mathrm{~cm}^{2} \mathrm{~V}^{-1} \mathrm{~s}^{-1}$ at low temperatures, far larger than for any other material, ${ }^{13}$ indicating potential applications in high-speed transistors and integrated circuits. The research group at IBM led by Phaedon Avouris has demonstrated SiC-based graphene field-effect transistors with a cutoff frequency over $300 \mathrm{GHz}$, thus setting a milestone in the development of nanoelectronics (see the article in this issue by Avouris and Xia).

All graphene growth techniques, including mechanical exfoliation of graphite, ${ }^{3}$ surface graphitization on $\mathrm{SiC}$, and catalytic graphitization on metal surfaces, ${ }^{5}$ are able to produce graphene samples with sizes up to tens of micrometers or larger. This advantage of large size has also enabled the measurement of novel nanoscale functionalities on single graphene structures.
The combination of the expected long spin lifetime with the high electron velocity related to their linear dispersion relation reveals the potential of graphene for spintronics (see the article in this issue by Seneor et al.).

Metrology is aimed at establishing definitions of universal units and implementing them in both the laboratory and industry. Improvements in electrical standards have so far come from solid-state quantum physics. Metrological investigations of the quantum Hall effect in graphene have demonstrated that a quantum resistance standard more robust than a GaAs-based one and able to benefit from recent helium-free cryogenic techniques can be developed. The metrological study of the quantum Hall effect in graphene has been shown to be very useful for the development of materials and also industrial applications such as microelectronics (see the article in this issue by Schopfer and Poirier).

Compared to other nanomaterials, one-atom-thick graphene sheets with a two-dimensional planar geometry can enhance electron transport for more effective devices for energy applications. Tremendous progress has been achieved in developing high-performance energy-conversion (e.g., solar cells and fuel cells) and energy-storage (e.g., supercapacitors and batteries) devices based on graphene materials (see the article in this issue by Liu et al.). Graphene has also shown unusual thermal properties, including very high in-plane thermal conductivity - strongly affected by interfacial interactions, atomic defects, and edges - and relatively low out-ofplane thermal conductance. In graphene composites and three-dimensional architectures, simulation results suggest that the thermal properties could be highly tunable. Such tunability raises the interesting prospect of ultrahigh thermal conductivity for heat-sink applications as well as ultralow thermal conductivity for thermoelectric applications (see the article in this issue by Pop et al.). 
The optical properties of graphene are also of great interest. The strong $\pi-\pi^{*}$ optical transitions in graphene and large domain sizes result in novel nonlinear optical properties with high laser damage threshold energy, broad-band absorption coefficients from UV to near IR spectra, and subpicosecond to microsecond responses by structural modifications (see the article in this issue by Cao et al.). With regard to the biological realm, graphene is a biocompatible material and could interface with biology for applications relating to electronics. Biosensing and bioimaging applications in enzyme biosensors, DNA biosensors, immunosensors, and cell imaging have all been explored (see the article in this issue by Yang et al.).

Many materials have multiple functional properties, so that the same material could be used in various applications. As mentioned previously, graphene has exhibited a long list of functionalities in electronics, photonics, nonlinear optics, thermal properties, terahertz applications, biofunctionality, composites, energy storage, and battery electrodes. Figure 3 illustrates a multilevel sketch of graphene science and one way of visualizing its functionalities. In the figure, the top level, inorganic chemistry and solid-state physics, represents the intellectual core of carbon science and technology. The chemistry and physics of graphene, governed by the unique graphitic hexagonal structures at the atomic scale, underpin the top level. The basic structural unit of graphene is a hexagonal ring consisting of $s p^{2}$ carbon atoms, which is the same as in other carbon materials and

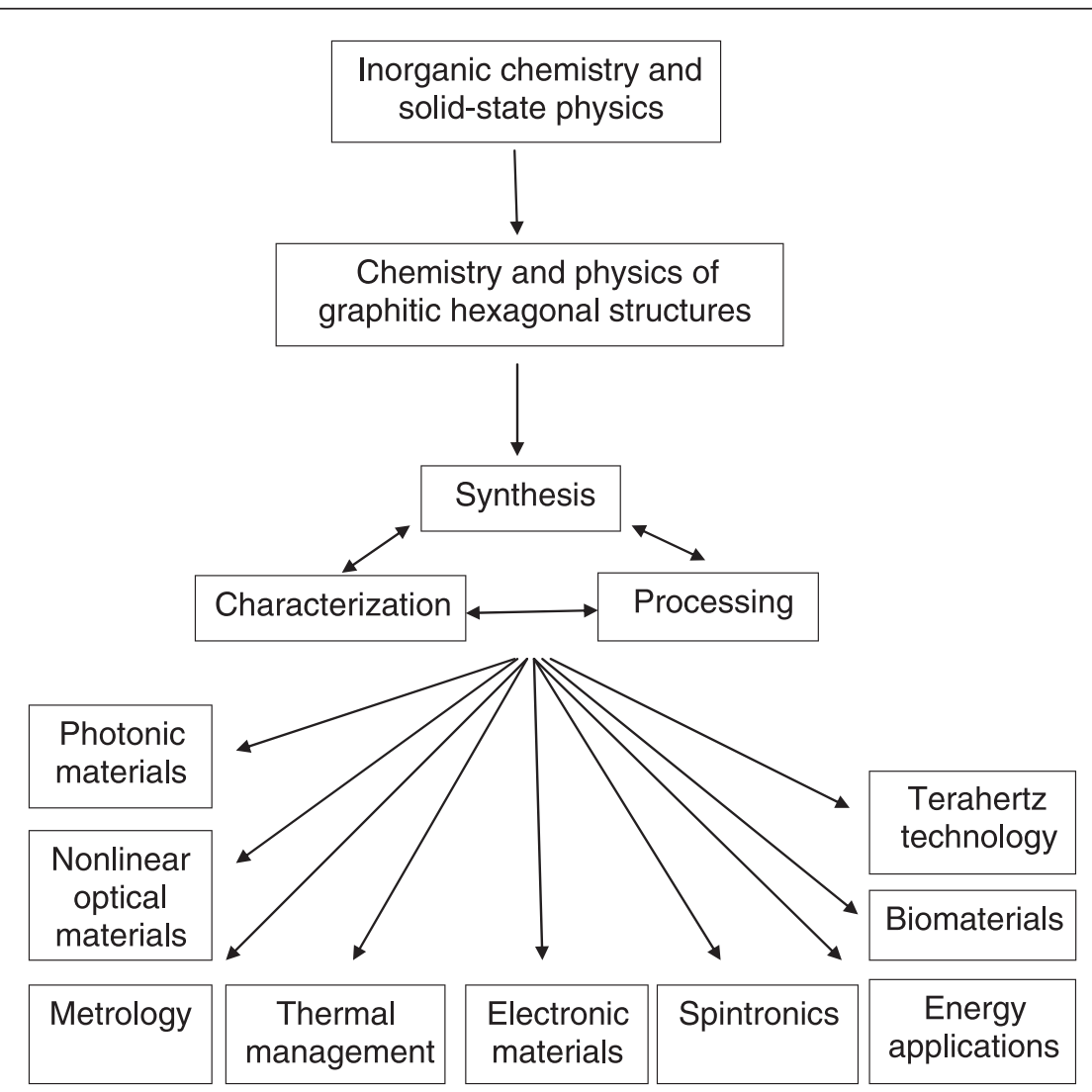

Figure 3. Multiple functionalities of graphene. structures such as graphite, carbon fibers, CNTs, and other highly graphitized types of carbon. The chemistry and physics of graphitic hexagonal structures represent the scientific fundamentals for developing graphene growth techniques and predicting various graphene functionalities. The middle level in Figure 3 is graphene synthesis, processing, and characterization. Several graphene growth and fabrication techniques have been developed, as outlined earlier in this article and detailed in this issue, and graphene materials have become a distinct material family. The methods of processing graphene for electronic devices and for biological sensors are very different, and various chemical groups are often applied to graphene to enhance specific functionality. The bottom level in Figure 3 represents the functionalities of graphene. Graphene-based materials exhibit different functionalities depending on the processing conditions and structures for different applications.

\section{Developments in graphene manufacturing technologies}

Whereas the multiple functionalities of graphene have been extensively explored, manufacturing techniques and commercialization are needed to utilize these functionalities of graphene; however, these remain in their infancy. An important lesson learned from the commercialization of CNTs is that the quality of the material is key for successful applications (see the article in this issue by Arthur et al.). Among the available graphene growth techniques, mechanical exfoliation from graphite is able to produce the highest-quality graphene when the best-quality graphite is used, but this technique is inefficient and does not appear to be a large-scale graphene production technique for graphene commercialization. Graphene production techniques involving chemical exfoliation, catalytic CVD on a metal surface, and $\mathrm{SiC}$ surface decomposition are being developed for commercialization (see the articles in this issue by Avouris and Xia, Bartelt and McCarty, and Jaber-Ansari and Hersam).

Large amounts of research funding have been invested thus far in graphene research, and multiple functionalities of graphene have consequently been discovered for potential applications. The initial technological advances have generated high expectations for graphene commercialization. Issues such as cost, production scale, product quality, and marketing often result in "commercialization anxiety," as described in the article in this issue by Arthur et al.

Graphene might have two advantages for successful commercialization: (1) Large-scale production has been achieved by chemical exfoliation from graphite, and (2) low-productioncost techniques are currently available using metal catalytic CVD and chemical exfoliation. However, the requirements for graphene quality 
depend on the nature of the application. For example, electronic applications demand the highest-quality graphene with low defect densities, large sizes, and abrupt interfaces (see the article in this issue by Avouris and Xia). For optical applications, un-exfoliated graphitic particles and amorphous carbon result in scattering, and the purity of graphene is one of the primary concerns (see the article in this issue by Cao et al.). The multiple functionalities of graphene thus offer the opportunity to develop a wide range of growth techniques with specific applications in mind.

The main technical barrier to the commercialization of graphene is the need to develop diverse fabrication techniques. As graphene technologies are advancing to commercialization and large-scale manufacturing, biological interactions and safety of graphene materials are emerging issues. Graphene materials show several unique modes of interaction with biological molecules, tissues, and cells. They could be either benign or harmful, and this biological response will vary according to the physicochemical properties of the material and the biologically effective dose (see the article in this issue by Jachak et al.).

Finally, two pioneers of carbon nanomaterials, Rodney Ruoff and Mildred Dresselhaus, share their perspectives on the future of nanostructured carbon materials. Ruoff discusses graphene and also speculates on its role as a precursor or template for making other materials and how those materials might be useful. Graphene has interesting possibilities as a building block for new materials, such as ultrathin $s p^{3}$-bonded large sheets, and as a type of structural analog of negative curvature carbon, which also has atom thick walls of trivalently bonded carbon. Dresselhaus briefly reviews nanostructured carbon from a historical perspective, discusses new developments to explore other layered materials and superlattice structures, and emphasizes the importance of scientific progress to address critical society needs.

\section{Conclusions}

Graphene has stimulated a new era of materials research in low-dimensional nanomaterials. The two-dimensional nature of graphene, with single-atom thickness, and its lateral size at the micrometer scale have opened up opportunities for the measurement of unique physical properties. The multiple functionalities of graphene continue to enrich many aspects of nanoscience and nanoengineering. The collection of novel techniques for growth and processing is anticipated to lead to the production of high-quality graphene for manufacturing and commercialization. Developing a fundamental understanding of the growth mechanisms of low-dimensional structures remains a major challenge and a significant opportunity for materials researchers.

\section{Acknowledgment}

We thank Steven C. Moss (The Aerospace Corporation) and Paul S. Drzaic (Apple Inc.) for helpful discussions. W.L. and J.B. acknowledge support from AFOSR (Charles Lee).

\section{References}

1. H.W. Kroto, J.R. Heath, S.C. Obrien, R.F. Curl, R.E. Smalley, Nature 318, 162 (1985).

2. S. lijima, Nature 354, 56 (1991).

3. K.S. Novoselov, A.K. Geim, S.V. Morozov, D. Jiang, Y. Zhang, S.V. Dubonos, I.V. Grigorieva, A.A. Firsov, Science 306, 666 (2004).

4. C. Berger, Z. Song, T. Li, X. Li, A.Y. Ogbazghi, R. Feng, Z. Dai, A.N. Marchenkov, E.H. Conrad, P.N. First, W.A. de Heer, J. Phys. Chem. B 108, 19912 (2004).

5. V. Singh, D. Loung, L. Zhai, S. Das, S.I. Khondaker, S. Seal, Prog. Mater. Sci. 56, 1178 (2011)

6. J. Haas, W.A. de Heer, E.H. Conrad, J. Phys.: Condens. Matter 20, 323202 (2008)

7. M.J. Allen, V.C. Tung, R.B. Kaner, Chem. Rev. 110, 132 (2010)

8. S.D. Sarma, S. Adam, E.H. Hwang, E. Rossi, Rev. Mod. Phys. 83, 407 (2011).

9. P.T. Araujo, M. Terrones, M.S. Dresselhaus, Mater. Today 15, 98 (2012).

10. M.S. Fuhrer, C.N. Lau, A.H. MacDonald, MRS Bull. 35, 289 (2010).

11. T. Kuilla, S. Bhadra, D. Yao, N.H. Kim, S. Bose, J.H. Lee, Prog. Polym. Sci. 35, 1350 (2010).

12. D.B. Fischbach, in Chemistry and Physics of Carbon, P.L. Walker Jr., Ed (Marcel Dekker, New York, 1971), vol. 7, p. 1.

13. S.V. Morozov, K.S. Novoselov, M.L. Katsnelson, F. Schedin, D.C. Elias, J.A. Jaszczak, A.K. Geim, Phys. Rev. Lett. 100, 016602 (2008).

14. P. Thrower, personal communication (2012).

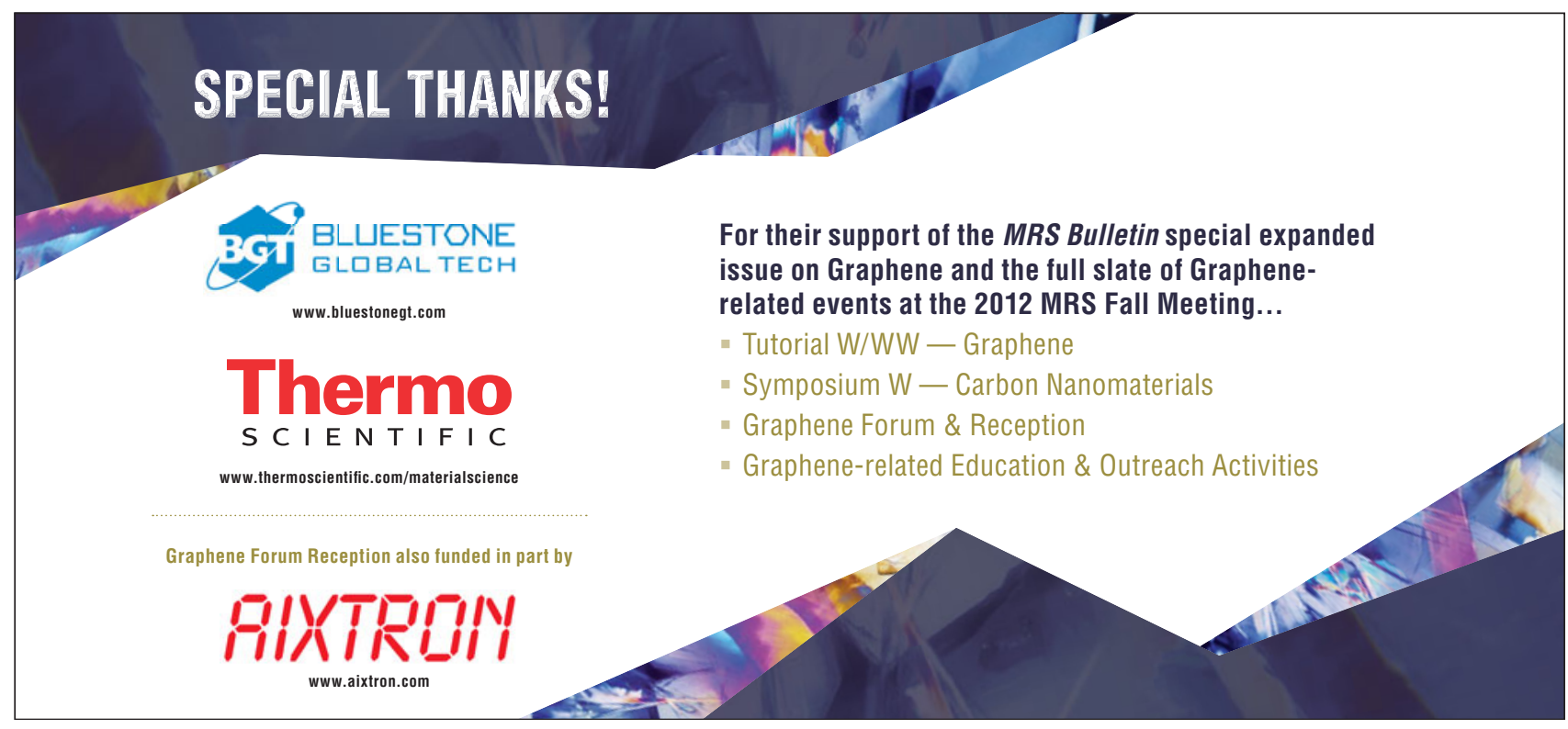

